\section{Effects of Genetic Engineering - The Ethical and Social Implications}

\author{
Satyajit Patra and \\ Araromi Adewale Andrew \\ American International Medical \\ University, Bosejour Road, Saint Lucia, \\ WI
}

Corresponding author: Satyajit Patra

dr.patra@aimu-edu.us

American International Medical University, Bosejour Road, Saint Lucia, WI

\begin{abstract}
Several works have been done on genetic engineering with major focus on its importance ranging from increasing plant and animal food production, diagnosing disease condition, improve medical treatment, and produce vaccines and other useful drugs. Methods in the techniques involve the selective breeding of animals and plants, hybridization (reproduction between different strains or species), and recombinant deoxyribonucleic acid (rDNA). However, these benefits are not without peril. This article gives a brief account on the effect of this principle on the biosphere together with several controversial issues that accompany the acceptance of this technology.
\end{abstract}

Public reaction to the use of rDNA in genetic engineering has been mixed. The production of medicines through the use of genetically altered organisms has generally been welcomed. However, critics of rDNA fear that disease-producing, organisms used in some rDNA experiments might develop extremely infectious forms that could cause worldwide epidemics [1].

Animal rights groups have argued that the production of transgenic animals is harmful to other animals. A related concern is that engineered fish for example may compete with wild fish for food and replace wild fish in some areas. Critics have questioned the safety of genetically engineered bovine somatotropin (BST) to increase the milk yield of dairy cows BST for both the cows that are injected with it and the humans who drink the resulting milk; owing to the fact that it increases a cow's likelihood of developing mastitis, or infection of the udder, and it also makes cows more susceptible to infertility and lameness. Nevertheless, the scientists consider the milk obtained from cows injected with
BST to be safe for human consumption [2].

Transgenic plants also present controversial issues. Allergens can be transferred from one food crop to another through genetic engineering. Another concern is that pregnant women eating genetically modified products may endanger their offspring by harming normal fetal development and altering gene expression [3]. Furthermore, the impact of this process on the environment cannot be over emphasized. Environmentalists have also argued that, due to natural selection, insects quickly develop resistance to plants that have been engineered to incorporate biological pesticides [4].

In 2002 the National Academy of Sciences released a report calling for a legal ban on human cloning. The report concluded that the high rate of health problems in cloned animals suggests that such an effort in humans would be highly dangerous for the mother and developing embryo and is likely to fail. Beyond safety, the possibility of cloning humans also raises a variety of social issues like the psychological issues that would result for a cloned child who is the identical twin of his or her parent [5].

Genetic engineering and transgenics continue to present intriguing and difficult challenges for $21^{\text {st }}$ century scientists and ethicists, and education and meaningful, respectful discourse are just the starting point of what is required to tackle such complex ethical issues. We can expect intense cross-disciplinary debate and discussion as new life forms are created through science and medicine. 


\section{References}

1 Fleischmann WR (1996) Viral Genetics, in Medical Microbiology. In: Baron S (ed.) University of Texas Medical Branch at Galveston: Galveston (TX).

2 Mepham TB (2000) The role of food ethics in food policy. Proc Nutr Soc 59: 609-618.

3 Smith JM (2003) Seeds of Deception: Exposing industry and government lies about the safety of the genetically engineered foods you're eating. 2003, Fairfield, IA: Yes! Books. 304.

4 D'Halluin K, Ruiter R (2013) Directed genome engineering for genome optimization. Int J Dev Biol 57: 621-627.

5 Powledge TM (2002) Will they throw the bath water out with the baby? The US Congress is still debating whether to outlaw cloning humans. EMBO Rep 3: 209-211. 